PROCEEDINGS OF THE

AMERICAN MATHEMATICAL SOCIETY

Volume 129, Number 10, Pages 3085-3090

S 0002-9939(01)05925-1

Article electronically published on April 2, 2001

\title{
SOME DESCRIPTIVE SET-THEORETIC PROPERTIES OF THE ISOMORPHISM RELATION BETWEEN BANACH SPACES
}

\author{
ANDRZEJ KOMISARSKI
}

(Communicated by Alan Dow)

\begin{abstract}
Consider the space $\mathcal{V}(E)$ of closed linear subspaces of a separable Banach space $E$ equipped with the standard Effros Borel structure. The isomorphism relation between Banach spaces being elements of $\mathcal{V}(E)$ determines a partition of $\mathcal{V}(E)$. In this note we prove a result describing the complexity of analytic subsets of $\mathcal{V}(E)$ intersecting a large enough number of the abovementioned parts of $\mathcal{V}(E)$.
\end{abstract}

\section{INTRODUCTION}

Given a separable Banach space $E$, we shall denote by $\mathcal{V}(E)$ the collection of closed linear subspaces of $E$ equipped with the Effros Borel structure. The Effros structure is the $\sigma$-algebra generated by the sets $\{F \in \mathcal{V}(E): F \cap U \neq \emptyset\}, U$ being open in $E$. The Effros Borel space $\mathcal{V}(E)$ is standard, i.e., it is Borel isomorphic to the unit interval $I$; cf. $[\mathrm{Chr}$, [Ke].

Recall that a set in a finite product $\mathcal{V}(E)^{m}$ is analytic if it is the projection of a Borel set in $\mathcal{V}(E)^{m} \times I$.

Bossard $[\mathrm{Bo}$ investigated descriptive set-theoretic properties of the isomorphism relation on $\mathcal{V}(E)$ (we call Banach spaces $X$ and $Y$ isomorphic if $X$ is linearly homeomorphic to $Y$; cf. [L-T]). Bossard proved that the isomorphism relation is analytic in $\mathcal{V}(E) \times \mathcal{V}(E)$ and, if $E$ is universal, the relation is not Borel and admits no analytic selectors ([Bo], Theorem 2).

The aim of this note is to prove the following.

1.1. Theorem. Let $\mathcal{A}$ be an analytic set in the Effros Borel space $\mathcal{V}(E)$ such that each Banach space of continuous functions on a countable compact topological space has an isomorphic copy in $\mathcal{A}$. Then $\mathcal{A}$ contains $2^{\aleph_{0}}$ pairwise isomorphic elements which are isomorphic to a Banach space of continuous functions on some countable compact space.

The result, strengthening Bossard's theorem on selectors, is related to some topics concerning Lusin's constituents. Let us comment on this aspect of the subject. Let $2^{\mathbb{Q}}$ be the Cantor set of all subsets of the rationals $\mathbb{Q}$ (identified with the characteristic functions), and let us consider in $2^{\mathbb{Q}} \times 2^{\mathbb{Q}}$ the relation of having the same order type. The equivalence class of a well-ordered set of type $\alpha$ is the $\alpha$ th Lusin

Received by the editors July 28, 1999 and, in revised form, March 5, 2000.

2000 Mathematics Subject Classification. Primary 03E15; Secondary 46 B03.

(C)2001 American Mathematical Society 
constituent; cf. [Ku], $\mathrm{Ke}$. V. G. Kanovei $\mathrm{Ka}$, answering an old question of Lusin, demonstrated that no analytic set in $2^{\mathbb{Q}}$ can hit every Lusin constituent in exactly one point (cf. CGP for a simpler proof). Analogous facts concerning the CantorBendixson derivative were discussed in [Ch-P]. Theorem [1.1] discloses a similar phenomenon for the isomorphism relation between Banach spaces with separable dual (note that this collection forms a coanalytic but not Borel set in $\mathcal{V}(E)$, provided $E$ is universal; see [Bo], Corollaire 7 , and $[\mathrm{Ke}], 33.24)$.

In fact, our proof of Theorem 1.1 given in section 3, will be based on the results about the Cantor-Bendixson derivative. In section 2 we shall clarify some notions and establish some background needed in this proof.

\section{Terminology AND SOME BACKGROUnd}

Our terminology concerning descriptive set theory follows Kechris $\mathrm{Ke}$ and $\mathrm{Ku}$ ratowski $[\mathrm{Ku}$. For the material related to Banach spaces and ordinals we refer to Semadeni $[\mathrm{Se}$. Some additional facts about ordinals we shall use can be found in [K-M].

2.1. Borel spaces and analytic sets. A Borel (or measurable) space is a pair $(A, \mathcal{S})$, where $A$ is an arbitrary set and $\mathcal{S} \subset 2^{A}$ is a $\sigma$-algebra of subsets of $A$. The elements of $\mathcal{S}$ are called Borel sets. We usually denote a Borel space $(A, \mathcal{S})$ just as $A$. In this case a $\sigma$-algebra $\mathcal{S}$ is assumed to be already defined or is not important. A Borel space $(A, \mathcal{S})$ is standard if there exists a separable completely metrizable topology on $A$, such that the Borel sets generated by this topology are exactly the elements of $\mathcal{S}$. According to this definition every topological space can be regarded as a Borel space and every separable complete metric space can be regarded as a standard Borel space. Every Borel subset of a standard Borel space equipped with a relative Borel structure is a standard Borel space.

A map $f: A \rightarrow B$ between Borel spaces $(A, \mathcal{S})$ and $(B, \mathcal{T})$ is Borel if $f^{-1}(T) \in \mathcal{S}$ for $T \in \mathcal{T}$.

A set $K \subset B$ is analytic in a standard Borel space $(B, \mathcal{T})$ if it is the projection of a Borel set in $(B \times I, \mathcal{T} \otimes \mathcal{B})$, where $I$ is a unit interval and $\mathcal{T} \otimes \mathcal{B}$ is the $\sigma$-algebra generated by the rectangles $T \times B$ with $T \in \mathcal{T}$ and $B$ open in $I$.

2.2. The Effros Borel space $\mathcal{V}(E)$. We shall fix some notation and mention a few basic facts concerning the space of closed linear subspaces of a Banach space and its Borel structure.

Let $E$ be a separable Banach space. We shall denote by $\mathcal{F}(E)$ the Borel space of nonempty closed subsets of $E$. Borel structure of $\mathcal{F}(E)$ is an Effros structure and is defined as a $\sigma$-algebra generated by sets of the form

$$
B_{U}=\{F \in \mathcal{F}(E): F \cap U \neq \emptyset\},
$$

where $U$ is an open subset of $E$. It is well known that $\mathcal{F}(E)$ is a standard Borel space. In $\mathcal{F}(E)$ we can consider a subset $\mathcal{V}(E)$ consisting of closed Banach subspaces of $E . \mathcal{V}(E)$ is Borel in $\mathcal{F}(E)$; hence $\mathcal{V}(E)$ equipped with the relative Borel structure is a standard Borel space. If $E$ is a Banach algebra with a unit we shall also consider the subset $\mathcal{H}(E) \subset \mathcal{V}(E)$ consisting of subalgebras with a unit. The set $\mathcal{H}(E)$ is Borel in $\mathcal{F}(E)$ and is a standard Borel space (after equipping with the relative Borel structure). Our main area of interest is the Banach algebra $C\left(2^{\mathbb{N}}\right)$ (i.e., the space of continuous functions on the Cantor set), so denote $\mathcal{F}=\mathcal{F}\left(C\left(2^{\mathbb{N}}\right)\right), \mathcal{V}=\mathcal{V}\left(C\left(2^{\mathbb{N}}\right)\right)$ and $\mathcal{H}=\mathcal{H}\left(C\left(2^{\mathbb{N}}\right)\right)$. 
The Banach space $C\left(2^{\mathbb{N}}\right)$ is universal in the class of separable Banach spaces, i.e., $C\left(2^{\mathbb{N}}\right)$ is separable and for every separable Banach space $E$ there exists a closed subspace in $C\left(2^{\mathbb{N}}\right)$ isomorphic to $E$. Therefore, the Borel space $\mathcal{V}(E)$ can be considered as a Borel subspace of $\mathcal{V}$.

2.3. The Cantor-Bendixson derivative and the Cantor-Bendixson rank. For any separable metric space $X$ the hyperspace $\mathcal{K}(X)$ is the space of compact subsets of $X$ with the Hausdorff metric; cf. $[\mathrm{Ke}$. If $X$ is a separable complete metric space, so is $\mathcal{K}(X)$. We shall denote by $\mathcal{K}$ the hyperspace $\mathcal{K}\left([-1,1]^{\mathbb{N}}\right)$ of the Hilbert cube.

The Cantor-Bendixson derivative of $K \in \mathcal{K}(X)$ is the set $K^{\prime}$ of all accumulation points of $K$. If $\xi$ is an ordinal, we define the $\xi$ th iterated Cantor-Bendixson derivative by the transfinite induction: $D^{0}(K)=K, D^{\xi}(K)=\left(D^{\zeta}(K)\right)^{\prime}$ if $\xi=\zeta+1$ and $D^{\xi}(K)=\bigcap_{\zeta<\xi} D^{\zeta}(K)$ if $\xi$ is a limit ordinal.

The Cantor-Bendixson rank on a hyperspace $\mathcal{K}(X)$ is a map $\delta_{C B}: \mathcal{K}(X) \rightarrow$ $\omega_{1} \cup\{\infty\}$, such that $\delta_{C B}(K)$ is the minimal ordinal $\xi$ with $D^{\xi+1}(K)=\emptyset$, or $\delta_{C B}(K)=\infty$ if $D^{\xi}(K) \neq \emptyset$ for every ordinal $\xi$.

We shall denote by $\mathcal{K}_{<\infty}$ the collection of countable subsets of the Hilbert cube, i.e., $\mathcal{K}_{<\infty}=\left\{K \in \mathcal{K}: \delta_{C B}(K)<\infty\right\}$.

2.4. The ordinals. We shall denote the minimal infinite ordinal by $\omega$, and the minimal uncountable ordinal by $\omega_{1}$. As usual, an ordinal $\xi$ will be identified with the segment $\{\alpha: \alpha<\xi\}$, and the segment will be considered with the order topology. By the Mazurkiewicz theorem, each countable compact space $K$ is homeomorphic to an ordinal $\omega^{\xi} n+1$, with $\xi$ countable and $n$ natural, and the CantorBendixson rank of $K$ is then equal to $\xi$, while $D^{\xi}(K)=n$; cf. [Se].

We shall need a theorem of Bassaga and Pełczyński B-Pe providing an isomorphical classification of the Banach spaces $C(\xi+1)$ of continuous functions on countable ordinals.

2.4.1. Theorem. For any two countable infinite ordinals $\xi$ and $\zeta$ Banach spaces $C(\xi+1)$ and $C(\zeta+1)$ are isomorphic if and only if $\xi<\zeta^{\omega}$ and $\zeta<\xi^{\omega}$.

Moreover, for any infinite $\xi<\omega_{1}$ the space $C(\xi+1)$ has dual $l_{1}$.

A set of ordinals $A \subset \omega_{1}$ is called a club if it is closed and unbounded in $\omega_{1}$. An intersection of countably many clubs is a club.

Let $\Lambda \subset \omega_{1}$ be the club of fixed points of the function $\xi \mapsto \omega^{\xi}$, i.e., $\Lambda=\{\xi<$ $\left.\omega_{1}: \xi=\omega^{\xi}\right\}$; cf. [K-M. If $\lambda \in \Lambda, \xi<\lambda$ and $n \in \mathbb{N}$, then $\left(\omega^{\xi} n\right)^{\omega} \leq\left(\omega^{\xi+1}\right)^{\omega}=$ $\omega^{(\xi+1) \omega} \leq \omega^{\omega^{\xi+1} \omega}=\omega^{\omega^{\xi+2}} \leq \omega^{\omega^{\lambda}}=\omega^{\lambda}$. Therefore, by Theorem 2.4.1 we get

2.4.2. Corollary. For any $\lambda \in \Lambda, \xi<\lambda$ and $n \in \mathbb{N}$ the Banach space $C\left(\omega^{\lambda}+1\right)$ is not isomorphic to $C\left(\omega^{\xi} n+1\right)$.

2.5. A result concerning the Cantor-Bendixson rank (cf. $\overline{\text { Ch-P }}$ ). We shall use the following fact concerning the Cantor-Bendixson rank $\delta_{C B}: \mathcal{K}(X) \rightarrow \omega_{1} \cup$ $\{\infty\}$; cf. section 2.3. The fact is a simple corollary of the main result in [Ch-P].

2.5.1. Theorem. For any metrizable compact space $X$ and any separable completely metrizable space $T$ let $\delta: T \times \mathcal{K}(X) \rightarrow \omega_{1} \cup\{\infty\}$ be a function defined by

$$
\delta(t, K)=\delta_{C B}(K) \text { for } t \in T \text { and } K \in \mathcal{K}(X),
$$


where $\delta_{C B}$ is the Cantor-Bendixson rank on $\mathcal{K}(X)$. Let $A \subset T \times \mathcal{K}(X)$ be an analytic set satisfying $A \cap \delta^{-1}(\{\xi\}) \neq \emptyset$ for $\xi$ in some club in $\omega_{1}$. Then, for all $\xi$ in some club, any $F_{\sigma^{-}}$set containing $A \cap \delta^{-1}(\{\xi\})$ intersects $A \cap \delta^{-1}(\{\alpha\})$ for some $\alpha<\xi$.

\section{Proof of Theorem 1.1}

Before proving the main theorem let us make a few observations.

First observe that it is enough to prove Theorem 1.1 with $E=C\left(2^{\mathbb{N}}\right)$. Indeed, let $E$ be arbitrary and let $\mathcal{A} \subset E$ satisfy the assumption of Theorem[1.1. We can embed $E$ into $C\left(2^{\mathbb{N}}\right)$ as a closed subspace. Then $\mathcal{V}(E)$ is Borel in $\mathcal{V}=\mathcal{V}\left(C\left(2^{\mathbb{N}}\right)\right)$ and $\mathcal{A}$ is analytic in $\mathcal{V}$. This reduces our problem to the case $E=C\left(2^{\mathbb{N}}\right)$.

We shall define a Borel map $\Phi: \mathcal{H} \rightarrow \mathcal{K}$, such that for any $H \in \mathcal{H}$ the algebra $C(\Phi(H))$ is isometric to $H$. To this end we shall apply the Kuratowski-RyllNardzewski theorem (cf. [Ke, Theorem 12.13) which allows one to select for each $H \in \mathcal{H}$ a collection of elements $d_{n}^{H} \in H, n \in \mathbb{N}$, dense in the unit ball of $H$ such that each map $d_{n}: \mathcal{H} \rightarrow C\left(2^{\mathbb{N}}\right)$ is Borel. (Note that the map $\mathcal{H} \rightarrow \mathcal{F}$ assigning to a closed subalgebra of $C\left(2^{\mathbb{N}}\right)$ its intersection with the closed unit ball in $C\left(2^{\mathbb{N}}\right)$ is Borel.)

The map $\Phi$ is defined as follows:

$$
\Phi(H)=\left\{\left(d_{1}^{H}(x), d_{2}^{H}(x), \ldots\right): x \in 2^{\mathbb{N}}\right\} .
$$

Obviously $\Phi(H)$ is a non-empty closed subset of the Hilbert cube $[-1,1]^{\mathbb{N}}$, for $H \in \mathcal{H}$.

3.1. Lemma. For any $H \in \mathcal{H}$, the algebra $C(\Phi(H))$ is isometrically isomorphic to the algebra $H$.

Proof. For any $h \in H$ and $y \in \Phi(H)$ let $\psi(h)(y)=h\left(\left(d_{1}^{H}, d_{2}^{H}, \ldots\right)^{-1}(y)\right)$. It is easy to see that $\psi: H \rightarrow C(\Phi(H))$ is well defined and it is a norm preserving homomorphism of the algebras. To check that it is surjective observe that $\psi(H)$ contains constant functions and that $\psi(H)$ separates the elements of $\Phi(H)$, so $\psi(H)=C(\Phi(H))$, by the Stone-Weierstrass theorem.

3.2. Remark. As a matter of fact the space $\Phi(H)$ is homeomorphic to a quotient space $2^{\mathbb{N}} / \simeq$, where $x_{1} \simeq x_{2}$ if and only if $h\left(x_{1}\right)=h\left(x_{2}\right)$ for all $h \in H$. Indeed, the map $\Phi$ is identifying exactly these points of Cantor set $2^{\mathbb{N}}$ which are in relation $\simeq$ and it is easy to verify that a topology of $\Phi(H)$ is a topology of quotient space.

3.3. Lemma. The map $\Phi: \mathcal{H} \rightarrow \mathcal{K}$ is Borel.

Proof. The $\sigma$-algebra of Borel sets in $\mathcal{K}$ is generated by the sets $B_{U_{1}, \ldots, U_{k}}=\{K \in$ $\left.\mathcal{K}: K \cap\left(U_{1} \times \ldots \times U_{k} \times[-1,1]^{\mathbb{N}}\right) \neq \emptyset\right\}$, where $k \in \mathbb{N}$ and $U_{1}, \ldots, U_{k}$ are open in $[-1,1]$. Let us fix a countable dense subset $M$ of $2^{\mathbb{N}}$. Then

$$
\begin{aligned}
\Phi^{-1}\left(B_{U_{1}, \ldots, U_{k}}\right) & =\left\{H \in \mathcal{H}: \exists_{x \in 2^{\mathbb{N}}} d_{1}^{H}(x) \in U_{1}, \ldots, d_{k}^{H}(x) \in U_{k}\right\} \\
& =\left\{H \in \mathcal{H}: \exists_{x \in M} d_{1}^{H}(x) \in U_{1}, \ldots, d_{k}^{H}(x) \in U_{k}\right\} \\
& =\bigcup_{x \in M}\left\{H \in \mathcal{H}: d_{1}^{H}(x) \in U_{1}, \ldots, d_{k}^{H}(x) \in U_{k}\right\} \\
& =\bigcup_{x \in M} \bigcap_{i=1}^{k}\left\{H \in \mathcal{H}: d_{i}^{H}(x) \in U_{i}\right\} .
\end{aligned}
$$


The sets $\mathcal{U}_{i, x}=\left\{H \in \mathcal{H}: d_{i}^{H}(x) \in U_{i}\right\}$ are Borel because, for any fixed $x \in M$, $\mathcal{U}_{i, x}$ is the preimage of $U_{i}$ under the composition of the Borel map $H \mapsto d_{i}^{H}$ and the evaluation map $f \mapsto f(x)$. Therefore, $\Phi^{-1}\left(B_{U_{1}, \ldots, U_{k}}\right)$ is Borel as a countable sum of finite intersections of Borel sets $\mathcal{U}_{i, x}$.

The Borel space $\mathcal{V}$ is standard and we can fix a completely metrizable separable topology on $\mathcal{V}$ such that the Borel structure of $\mathcal{V}$ is the $\sigma$-algebra generated by the open sets. In the sequel, we shall refer to this topology. Let $\mathcal{R} \subset \mathcal{V} \times \mathcal{V}$ be the collection of pairs of Banach subspaces of $C\left(2^{\mathbb{N}}\right)$ that are isomorphic. By the result of Bossard $[\mathrm{BO}, \mathcal{R}$ is an analytic set.

We shall define a rank $\delta: \mathcal{V} \times \mathcal{K} \rightarrow \omega_{1} \cup\{\infty\}$ by the formula

$$
\delta(L, K)=\delta_{C B}(K) \text { for } L \in \mathcal{V} \text { and } K \in \mathcal{K},
$$

where $\delta_{C B}: \mathcal{K} \rightarrow \omega_{1} \cup\{\infty\}$ is the Cantor-Bendixson rank on the hyperspace $\mathcal{K}$ of the Hilbert cube.

We can now pass to the proof of Theorem 1.1 .

Proof of Theorem 1.1 (the case $E=C\left(2^{\mathbb{N}}\right)$ ). Let $\mathcal{A}$ be an analytic set in $\mathcal{V}$ containing isomorphic copies of every separable Banach space of continuous functions on a countable compact topological space and let

$$
\widehat{\mathcal{A}}=(\mathcal{A} \times \mathcal{K}) \cap\left(\operatorname{Id}_{\mathcal{V}} \times \Phi\right)(\mathcal{R} \cap(\mathcal{V} \times \mathcal{H})),
$$

where $\operatorname{Id} \mathcal{V}$ is the identity on $\mathcal{V}$ and $\Phi$ is defined by (2). According to this definition and Lemmas 3.1 and 3.3 set $\widehat{\mathcal{A}}$ is analytic in $\mathcal{V} \times \mathcal{K}$, and it consists of pairs $(L, K) \in$ $\mathcal{V} \times \mathcal{K}$ such that $L \in \mathcal{A}, L$ is isomorphic to $C(K)$ and there exists $H \in \mathcal{H}$ satisfying $\Phi(H)=K$.

For any infinite $\xi \in \omega_{1}$ set $\mathcal{A}$ contains an element $L_{\xi}$ isomorphic to $C\left(\omega^{\xi}+1\right)$ and there exists an algebra $H_{\xi} \in \mathcal{H}$, such that $\Phi\left(H_{\xi}\right)$ is homeomorphic to $\omega^{\xi}+1$. To see that define $H_{\xi}$ as $\left\{f \circ h: f \in C\left(\omega^{\xi}+1\right)\right\}$, where $h: 2^{\mathbb{N}} \rightarrow \omega^{\xi}+1$ is a continuous surjection and apply Remark 3.2 We have $\left(L_{\xi}, \Phi\left(H_{\xi}\right)\right) \in \widehat{\mathcal{A}}$ and $\delta\left(L_{\xi}, \Phi\left(H_{\xi}\right)\right)=\xi$. This implies that for any infinite $\xi \in \omega_{1}$ the intersection $\widehat{\mathcal{A}} \cap \delta^{-1}(\{\xi\})$ is nonempty and we can apply Theorem 2.5.1 with $\widehat{\mathcal{A}}$ analytic in $\mathcal{V} \times \mathcal{K}$. The conclusion of Theorem 2.5.1 states that there exists a club $\Theta \subset \omega_{1}$ such that for any $\xi \in \Theta$ every $F_{\sigma}$-set containing $\widehat{\mathcal{A}} \cap \delta^{-1}(\{\xi\})$ intersects $\widehat{\mathcal{A}} \cap \delta^{-1}(\{\zeta\})$ for some $\zeta<\xi$.

We shall show that if $0<\xi \in \Lambda \cap \Theta$ (note that $\Lambda \cap \Theta$ is a club in $\omega_{1}$ and hence is uncountable), then $\mathcal{A}$ contains uncountably many isomorphic copies of $C\left(\omega^{\xi}+1\right)$. Aiming at a contradiction, assume that there exists $0<\xi \in \Lambda \cap \Theta$, such that $\mathcal{I}_{\xi}=\left\{L \in \mathcal{A}: L\right.$ is isomorphic to $\left.C\left(\omega^{\xi}+1\right)\right\}$ is countable. For every $K \in \mathcal{K}$ satisfying $\delta(K)=\xi$ the set $K$ is homeomorphic to $\omega^{\xi} n+1$ with $n<\omega$ and due to Theorem 2.4.1 $C(K)$ is isomorphic to $C\left(\omega^{\xi}+1\right)$. Hence the $F_{\sigma}$-set $\mathcal{I}_{\xi} \times \mathcal{K} \subset \mathcal{V} \times \mathcal{K}$ contains $\widehat{\mathcal{A}} \cap \delta^{-1}(\{\xi\})$ and it must intersect $\widehat{\mathcal{A}} \cap \delta^{-1}(\{\zeta\})$ for some $\zeta<\xi$. The last statement means that there exists $L \in \mathcal{I}_{\xi}$ isomorphic to $C\left(\omega^{\zeta} n+1\right)$ with $n \in \mathbb{N}$ and consequently $C\left(\omega^{\xi}+1\right)$ is isomorphic to $C\left(\omega^{\zeta} n+1\right)$. This contradicts Theorem 2.4.2. The above contradiction proves that $\mathcal{I}_{\xi}$ is uncountable and since it is an analytic set in the standard Borel space $\mathcal{V}$ it contains $2^{\aleph_{0}}$ elements.

\section{REFERENCES}

[B-Pe] C. Bessaga, A. Pełczyński, Spaces of continuous functions (IV) (On isomorphical classification of spaces of continuous functions), Studia Math. 19 (1960), 53-62. MR 22:3971 
[Bo] B. Bossard, Codages des espaces de Banach séparables. Familles analytiques ou coanalytiques d'espaces de Banach, C. R. Acad. Sci. Paris Sér. I Math. 316 (1993), 1005-1010. MR 94g:46022

[CGP] J. Chaber, G. Gruenhage, R. Pol, On a perfect set theorem of A. H. Stone and N. N. Lusin's constituents, Fund. Math. 148 (1995), 309-318. MR 96k:54061

[Ch-P] J. Chaber, R. Pol, On the Cantor-Bendixson derivative, resolvable ranks and perfect set theorems of A. H. Stone, Israel J. Math. 110 (1999), 103-123. CMP 2000:11

[Chr] J. P. R. Christensen, Topology and Borel structure, North Holland, Amsterdam, 1974. MR 50:1221

[Ka] V. G. Kanovei, On uncountable sequences of sets determined by sieve operations, Dokl. Akad. Nauk SSSR 257 (1981), 808-812.

[Ke] A. S. Kechris, Classical descriptive set theory, Springer-Verlag, New York, 1995. MR 96e:03057

[Ku] K. Kuratowski, Topology, Vol. I, Państwowe Wydawnictwo Naukowe, Warsaw, 1966. MR 36:840

[K-M] K. Kuratowski, A. Mostowski, Set theory, North Holland, Amsterdam, 1976. MR 58:5230

[L-T] J. Lindenstrauss, L. Tzafriri, Classical Banach spaces, Springer-Verlag, Berlin, 1996.

[Se] Z. Semadeni, Banach spaces of continuous functions, Państwowe Wydawnictwo Naukowe, Warsaw, 1971. MR 45:5730

Department of Mathematics, Warsaw University, ul. Banacha 2, 02-097 Warsaw, POLAND

E-mail address: andkom@mimuw.edu.pl 